\title{
Long Non-coding RNAs in Peripheral Blood Mononuclear Cells Associated with Alzheimer Disease
}

\author{
Serap Kurt (Corresponding author) \\ Department of Medical Biology and Genetics, Dokuz Eylul University \\ 35340 Narlidere, Balcova, Izmir, Turkey \\ E-mail: serap.kurt@ogr.deu.edu.tr \\ Ayse Gaye Tomatir \\ Department of Medical Biology, Pamukkale University \\ 20070 Kinikli, Pamukkale, Denizli, Turkey \\ E-mail: tomatir@pau.edu.tr
}

\begin{abstract}
Noncoding RNAs have been implicated in important roles in cellular processes and in various diseases with the discovery of novel RNAs. Non-coding RNAs are classified as two groups according to their size. Transcripts with a length of 18-25 nucleotides, including microRNAs (miRNAs), which are important classes that can control the expression of many genes are called as short non coding RNAs while RNAs that greater than 200 nucleotides are termed as long non-coding RNAs (lncRNAs). It was found that lncRNAs were able to regulate gene expression at transcriptional, post-transcriptional and epigenetic levels. Recently, many lncRNAs have been shown to regulate amyloid beta (A $\beta$ ) production and synaptic loss in neurons in the nervous system in Alzheimer's Disease (AD). AD is a neurodegenerative disease which is most common in elderly, characterized by amyloid beta plaque accumulation outside the cell, neurofibrillary tangles in the cell and neuronal loss in the nervous system. The definitive diagnosis of $\mathrm{AD}$ in the clinic can only be made by observing these pathological changes in the brain during postmortem period. Therefore, there is a great need for biomarkers that may allow the disease to be identified especially at an early stage. The lncRNAs, which are thought to contribute to the development of disease, are seen as both targets and tools in new treatment approaches. It is thought that new treatment approaches can be developed by illuminating the functions of all lncRNAs in human genome and it can be used as biomarkers in the early diagnosis of diseases. After the discovery that serum, plasma and mononuclear cells in the blood reflect inflammatory pathogenesis in search for a biomarker to be involved in the diagnosis of $\mathrm{AD}$, studies have focused on peripheral blood. Recent studies have shown that mononuclear cells (PBMC) found in peripheral blood reflect inflammatory and apoptotic mechanisms in AD more in comparison to serum and plasma-based biomarkers.
\end{abstract}

Keywords: Long non-coding RNA, Alzheimer disease, Peripheral Blood Mononuclear Cells

DOI: $10.7176 / \mathrm{JSTR} / 5-10-03$

\section{Introduction}

RNAs are known to play a role in important processes in cells. In particular, non-coding RNAs (98.8\% of the human genome) have been shown to be involved in cellular defense, developmental processes, differentiation, DNA replication, transcription, and post-transcriptional gene silencing (Tan et al 2013). Defects in ncRNAs play pivotal roles in the pathogenesis of many diseases such as cancers, neurodegenerative diseases, mitochondrial diseases, immunodeficiency diseases and cardiovascular diseases (Akkaya et al 2013; Perez et al 2014; Vencken et al 2015; Tao et al 2015; Gomes et al 2018). Recent studies have identified many miRNAs as efficient biomarkers in the pathogenesis of Alzheimer's Disease (AD). For example, Liu et al (2016) found that miR-106b inhibits tau protein phosphorylation which is effective in the pathogenesis of AD by targeting the fyn gene and Reddy et al (2017) identified 
miR-124 as downregulated in neurons and targeted to the BACE1 gene, which plays a key role in AD. Another study showed that miR-135b has a neuroprotective effect by targeting the amyloid precursor protein (APP) cut enzyme, which is also prominent in the pathogenesis of AD (Zhang et al. 2016). In another study, miR-133b has been shown to act as a new diagnostic biomarker for AD by targeting EGFR and may have a neuroprotective role in AD (Yang Q et al. 2019). Although many biomarkers of neurodegenerative diseases have been identified in studies with miRNAs, research with lncRNAs is limited. The fact that LncRNAs are expressed extensively in the genomic regions of the brain suggests that they may be directly or indirectly associated with these diseases. In several diseases such as major depression disease, multiple sclerosis, amylotrophic sclerosis and several cancers, several lncRNAs have been identified as biomarkers and have been proposed to be used in the diagnosis of the disease. (Santaro et al 2016; Huang et al 2016; Elkouris et al 2019; Prinz et al 2019). In studies investigating lncRNAs, it shows that it has great effects on gene expression, especially in the central nervous system (CNS). Some lncRNAs expressed in CNS have an effect on neuronal cell differentiation by processes such as chromatin rearrangement. More importantly, these lncRNAs have also been associated with neurodegenerative diseases, and several lncRNAs with high expression in AD have been identified. For example, lncRNA known as BACE1-AS, has been shown to up-regulate in AD by affecting the stabilization of BACE1 mRNA and contribute to disease-specific amyloid beta $42(\mathrm{~A} \beta-42)$ protein formation (Akkaya and Dinçer 2013). Alzheimer's disease (AD) is the most common neurodegenerative disease and accounts for 60$80 \%$ of all dementia cases (Ryan et al 2018). Histopathologically, AD composed of plaques of amyloid beta $(A \beta)$ peptides are characterized by neurofibrillary tangles formed by hyperphosphorylated tau protein forms, as well as neuronal loss in specific regions of the brain (Prendecki et al. 2019). The definitive diagnosis of AD can only be made by observing these pathological changes in the brain in the postmortem period (Lashley et al. 2018, Yang et al. 2018). For the diagnosis of "probable" AD, the criteria of Neurological and Communicative Disorders and Stroke and Alzheimer's Disease and Related Disorders Association (NINCDS/ADRDA), which have $81 \%$ sensitivity and $70 \%$ specificity, are used today and accurate diagnosis up to $85 \%$ by examination only (McKhann et al. 2011). As biomarkers of neurodegeneration, peripheral blood mononuclear cells (PBMCs) have recently received considerable attention. PBMCs are thought to share most of the nonsynaptic biochemical environment of neurons. Although there are many studies conducted with lncRNAs in PBMCs in literatüre review, no similar study was found in AD.

\section{Alzheimer's Disease}

$\mathrm{AD}$ is a progressive neurodegenerative disorder that results in irreversible loss of cognitive skills and memory. It is the most common cause of dementia in elderly (Luo et al 2016). The disease was first described in 1906 by German psychiatrist and neuropathologist Alois Alzheimer as "a strange disease of the cerebral cortex" and in the following year was published in the Journal of General Psychiatry and Forensic Medicine (Allgemeine Zeitschrift für Psychiatrie und Psychich-Gerichtliche Medizine). The characteristic signs of the disease "senile plaques and neurofibrillary tangles" were first described in here (Selekler 2010). AD leads to progressive destruction of neurons, leading to a decline in cognitive functions. It causes mental and physical behavior disorder during the illness, makes it difficult to maintain daily activities and leads to deterioration in social relations (Jazvinšćak et al 2018). Memory loss is the first and most prominent sign of cognitive impairment, followed by aphasia (acquired language disorder), agnosia (apathy), apraxia (speech disorder) and behavioral disorders. There is currently no treatment that can completely reverse the disease.

Amyloid Precursor Protein (APP) is an integral transmembrane protein whose function is not fully elucidated. $A \beta$ proteins, the main components of $A \beta$ plaques that play a key role in $A D$, are formed by proteolytic cleavage of APP. Proteolytic cleavage of APP with $\alpha, \beta$ and $\gamma$-secretase enzymes produces different types of peptides. Proteolysis of APP in the so-called "non-amyloidogenic pathway" is mostly caused by $\alpha$-secretases. $\alpha$-secretases form the extracellular protein called sAPP $\alpha=$ P3 which is non-toxic and soluble in the cytosol, by cutting the APP approximately in the middle. Neurotrophic positive effects of this molecule on neurons have been shown (Bird and Miller 2005). However, in the pathway called "amyloidogenic", which is seen especially in neurons, APP is broken down with $\beta$ and az- secretases, resulting in the production of $A \beta$ peptides of different sizes. $\beta$-secretases cut APP from the amino terminal and $\gamma$-secretases cut off the carboxy terminal to form insoluble A $\beta$ forms (Iwatsubo et al 1994). The $A \beta$ s formed are 40 or 42 amino acids in length and are symbolized as $A \beta-40$ and $A \beta-42$, respectively. $\mathrm{A} \beta-42$ is the predominant species found in amyloid plaques in the brain and is more likely to form 
aggregates (Van Cauwenberghe et al 2016). These proteins, which accumulate outside the cell as a result of cuts, turn into dense neuritic plaques (Cummings 2004).

Another factor in the pathogenesis of $\mathrm{AD}$ is intercellular neurofibrillary tangles (NFTs). The main components of NFTs are hyperphosphorylated tau proteins. The tau protein is encoded by the MAPT (microtubule-associated protein tau) gene on chromosome 17. The function of the tau protein is to ensure the stability of the microtubules and the integrity of the cell skeleton and also to perform axonal transport (Jazvinšćak et al 2018). It is known that abnormal phosphorylated tau proteins during neurodegeneration process disrupt the ability to bind to microtubules, causing defects in axonal transport and then polymerize into insoluble double-stranded filaments to form intercellular NFTs (Poorkaj et al 1998).

\section{LncRNAs and Their Biogenesis}

It is known that only less than $2 \%$ of the human genome contains transcripts and more than $80 \%$ is not translated into protein. Until recently, non-coding RNA (ncRNAs), defined as "junk" or "transcriptional noise", are now known to function in controlling signaling pathways (Chew et al 2018). In the human genome, ncRNA genes form functional RNA molecules without protein coding and act as regulators in regulatory processes that have key roles within the cell. The RNA world is a heterogeneous group of ribosomal RNA, transfer RNA, IncRNA, miRNA, circular RNA and other small RNA molecules (Figure 1). Length-based classification is commonly used to classify ncRNAs. RNAs smaller than 200 nucleotides, including microRNA (miRNA), small interfering RNA (siRNA), piwi-associated RNA (piRNA), are called short non-coding RNAs; longer transcripts are known as long non-coding RNA (Akkaya and Dinçer 2013; Viereck and Thum 2016).

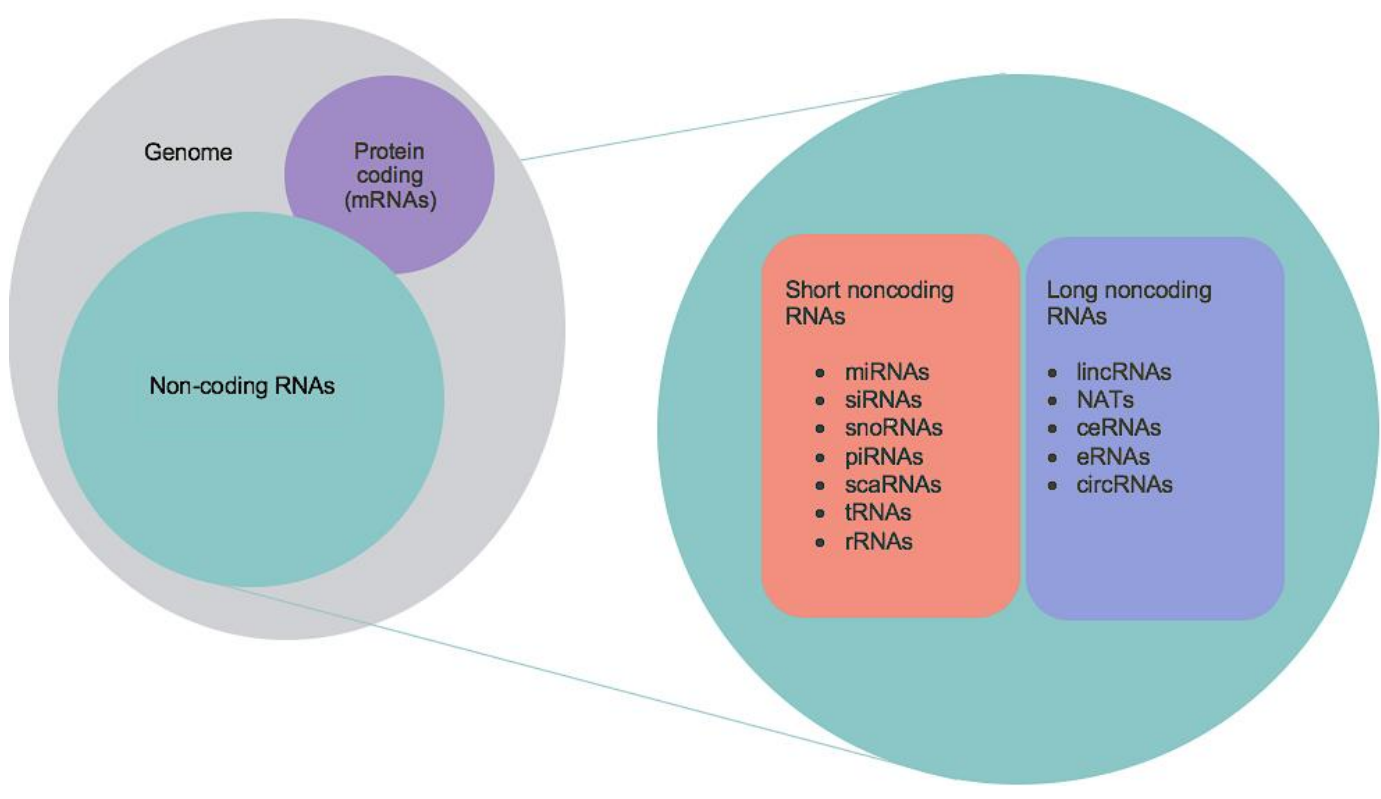

Figure 1. RNA classification based on lenght

LncRNAs have been shown to have critical functions in regulation of gene expression, cellular processes such as development and differentiation, and are able to regulate gene expression at the transcriptional, post-transcriptional, epigenetic level (Bayoğlu and Cengiz 2017). The functions of the vast majority are still unknown, but are thought to be involved in numerous biological processes. LncRNAs tend to be expressed at a very low level. Although most of them lack protein coding ability, it is known that very few lncRNAs also have protein coding potential (Schmitz et al 2016). In the current genome description studies, the number of lncRNA genes is around 16,000 in the human genome and in the mouse genome has been estimated to be around 9000 , but as the work continues, these numbers are likely to increase. In addition, the number of IncRNA transcripts exceeds the number of genes, since a gene may be template for several different splice variants of a lncRNA (Melissari and Grote 2016). 
An accepted classification system of lncRNAs is a system based on their location; intergenic, intronic, antisense chain or upstream of protein-coding genes are named according to their presence (Akman and Bensan 2014, Bär and Thum 2016). Sense RNAs are transcripts that overlap the coding gene within an intron on the same chain. Sense intronic RNAs; they are also localized within the intron of a coding gene but do not overlap with any exon. Antisense RNAs are RNAs that have transcripts that intersect any exon in the reverse lane of a protein-coding locus or have been proven to reverse regulation of a coding gene. Bidirectional RNAs are transcripts located in the opposite chain but located within $1 \mathrm{~kb}$ of the promoter on the sense chain. These are transcribed into the opposite direction of the promoter on the sense chain. LincRNAs (Long Intergenic RNAs) are transcripts that longer than 200 nucleotides and located between two coding genes. The IncRNAs have no biochemical differences from mRNAs, except that they do not contain an Open Reading Frame (ORF). However, they are shorter than mRNAs, able to exhibit ciseffective regulation, have less but longer exons, are expressed at relatively low levels, and exhibit a weaker profile in preserving primary sequences, indicating that they differ from mRNAs (Quinn and Chang 2016). LncRNAs can be located in the nucleus or in cytosolic fragments, polyadenylated or nonpolyadenylated, and often transcribed from both strands within a protein-coding locus (Tan et al 2013). They contain an average number of exons less than mRNAs and have lower expression levels in different tissues (Derrien et al. 2012).

The IncRNAs transcribed by Polymerase II, if necessary, 5'-cap addition, after the transcriptional arrangements such as pre-lncRNA splicing and polyadenylation, the maturation step involves the formation of a constant secondary (and tertiary) structure that gives the unique functional roles of IncRNA. (Ayers 2013).

The presence of 5'-cap positively affects lncRNA stability. A small proportion (about 15\%) of intronic RNAs without 5'-cap insertion is probably less stable because they form intron lariats in the cell. Despite the lack of coding potential, the majority of intronic and antisense lncRNAs reside in the nucleus and cytoplasm, suggesting their new role in the regulation and regulation of cytoplasmic processes (Ayupe et al 2015). Over $80 \%$ of known lncRNAs are localized in the nucleus (Kapranov et al. 2007), and for lncRNAs, their best-defined function in the nucleus is their role in regulating gene and genome activity at various levels (Schmitz et al. 2016). They participate in many processes including chromatin rearrangement, histone modifications, modification of genes by alternative splicing, and regulation of gene expression (Zhang et al. 2017). Together with the chromatin modifying complexes and various transcription regulators, they directly interact with DNA to regulate the expression of genes in the nucleus. Non-cytoplasmic coding lncRNAs can act as sponge for other transcripts or proteins such as miRNAs, serve as templates for the synthesis of small peptides, provide for degradation of mRNA or regulate translation (Viereck and Thum 2016). If the cellular function of a particular lncRNA is known exactly, they can be categorized according to their function, such as signal molecule, decoy, guide, enhancer, scaffold, molecular sponge or circular lncRNA. Since they can regulate gene expression at transcriptional or post-transcriptional levels, they may be localized in the nucleus or cytoplasm. In general, lncRNAs that control at the post-transcriptional level are lncRNAs that function as miRNA sponge by competing with miRNAs to bind to mRNA (Bär and Thum 2016).

LncRNAs can also interfere with protein translation by blocking, stabilizing or destabilizing mRNAs. LncRNAs localized in the nucleus, on the contrary, function as modulators of gene expression at the epigenetic and transcriptional level, regulating target genes "closely or remotely" in a cis or trans manner respectively (Batista and Chang 2013). Signal lncRNAs are the type of lncRNAs that are expressed only at a given place and time to send various alerts. Such expressed lncRNAs can interact with chromatinmodifying enzymes (eg histone methyltransferases) to provide silencing of target genes by transcriptional inhibition or by forming heterochromatin. Decoy lncRNAs indirectly suppress transcription by binding to regulatory factors such as transcription factors, chromatin remodels, or other RNA-binding proteins, thereby separating these factors from their specific targets. ceRNAs are examples of decoy lncRNAs. LncRNAs not only assist in the assembly of multiple ribonucleoprotein complexes, but can also function as functional components of these complexes themselves. They serve as molecular scaffolds. Guide lncRNAs bind to regulatory proteins and attract a ribonucleoprotein complex to their target site. These lncRNAs can act "cis or trans" effectively and mediate the activation or suppression of genes depending on whether the directed complexes are transcription factors. Enhancers are regulatory genomic elements that are too far from the promoter or transcriptional start site of the target genes. These lncRNAs are cisacting molecules produced from active enhancer elements and are required for activation of enhancer functions as well as the expression of adjacent coding genes (Bär and Thum 2016). LncRNAs have also 
been shown to regulate physiological processes such as inactivation of the X chromosome in mammals. It is known that lncRNA, called X-inactivation specific transcript (XIST), forms a polycomb complex to silence the $\mathrm{X}$ chromosome that performs its transcription. In contrast, another IncRNA, called TSIX, transcribes from the opposite chain of XIST and regulates the level of XIST during the inactivation process of X (Gayen et al 2016).

\section{LncRNAs Associated with Human Diseases}

The different expression of lncRNAs in diseases or the fact that any mutation in their structure directly or indirectly affects cellular processes has increased interest in understanding their functions and mechanisms of action (Ulitsky 2018). Recent studies have shown that lncRNAs are involved in tumorigenesis and tumor progression and also show metastatic features in various cancer conditions (Zhang et al. 2013). For example, Homeobox antisense intergenic RNA (HOTAIR) is known to be an important prognostic biomarker and identified as upregulated in gastric adenocarcinoma tissues. It has been suggested that increased HOTAIR expression is associated with high expression of the SUZ12 gene in these tissues and that these two may affect epigenetic regulation in gastric adenocarcinoma tumor tissues (Ayers 2013). HOTAIR is also known to incorporate chromotin-modifying protein complexes into the molecular scaffold structure (Akman and Bensan 2014) and overexpression of breast, colorectal, hepatocellular, gastrointestinal and pancreatic cancers has been reported (Beckedorff et al 2013).

LncRNAs include those involved in metastasis and tumor progression as oncogenes and tumorsuppressors, and regulating signaling pathways associated with these processes. Pregnancy-induced noncoding RNA (PINC) and prostate-specific transcript (PCGEM1) were the first oncogenic lncRNAs found to be overexpressed in breast and prostate carcinomas, respectively (Karaarslan and Serin 2016). However, positive effects of lncRNAs in some cancers have also been determined. One mechanism that cells can use to prevent tumor formation is oncogen-induced senescence (OIS). The INK4B-ARF-INK4A locus, which has a central role in OIS, is silenced in cells proliferated by Polycomb group proteins. This mechanism is dependent on lncRNA ANRIL, which is transcribed in the INK4B-ARF-INK4A locus and plays a cis-acting role. It is also known that the MIR31HG gene, located $400 \mathrm{~kb}$ above the INK4B-ARFINK4A locus, encodes another lncRNA involved in the regulation of INK4A (Schmitz et al 2016). LncRNAs are also play a role in tumor suppressor p53 and associated signaling pathways. p53 is known to bind to a large number of lncRNAs, such as linc -p21, which regulate gene expression in conjunction with p21 (Wu et al 2014). In addition, the main lncRNAs associated with some diseases are given in table 1.

Table 1. Identified lncRNAs in various diseases

\begin{tabular}{r|l} 
Disease & IncRNAs \\
Breast Cancer & GAS5, SNHG2, H19, Kcnq1ot1 \\
Gastric Cancer & GCAT1, H19, SUMO1P3, HOTAIR \\
Liver Cancer & HULC, HOTAIR, MALAT1 \\
Lung Cancer & MALAT1, TUG1, BANCR, GAS5 \\
Bladder Cancer & UCA1, H19, Linc-UBC1, MALAT1 \\
Melanoma & BANCR \\
Brain Tumors & Anti-NOS2A, MEG3 \\
Alzheimer's Disease & BACE1-AS \\
Spinocerebellar Ataxia & ATXN8OS \\
Fragile X Syndrome & FMRP \\
Lymphoma & RMRP \\
Neuroblastoma & MALAT1
\end{tabular}


Besides, lncRNAs are known to participate in epilepsy, schizophrenia and neurodegeneration process and play a role in many neurological diseases besides various cancers ( $\mathrm{Gu}$ et al. 2018). For example, a study performed in 2019, showed abnormal expression of sex-based lncRNAs in schizophrenia patients and their estimated utilizations as diagnostic and therapeutic tools (Fallah et al 2019). In addition, in AD, a study targeted to determine the regulatory role of lncRNA small nucleolar RNA host gene 1 (SNHG1) in A 25 -35-induced neuronal cell damage. The results showed that the knockdown of SNHG1 demonstrated neuronal protective effects by suppressing kringle containing transmembrane protein 1 (KRENEN1) by acting as a sponge for miR-137 in the AD in vitro cell model (Wang $\mathrm{H}$ et al. 2019). In another study conducted by Fotuhi et al in 2019, the expression levels of lncRNA BACE1-AS in plasma and plasma-derived exosomes of individuals with $\mathrm{AD}$ compared to healthy controls was investigated. As a result of the study, researhers found that BACE1-AS levels were lower in the pre-AD subgroup, but higher in subjects with full-AD compared to healthy controls. Therefore plasma lncrna BACE1-AS in patients with $\mathrm{AD}$ was suggested to be a suitable blood-based biomarker for $\mathrm{AD}$ diagnosis (Fotuhi et al. 2019). BC200 is known to be upregulated in AD. Therefore, in one study, assuming that inhibition of BC200 by siRNA is an effective method for the treatment of the disease, an AD cell model overexpressing A $\beta 1-42$ was created to investigate the effects of $\mathrm{BC} 200$ on cell viability and apoptosis and the associated mechanisms. Indeed, the results have shown that BC200 regulates AD cell viability and apoptosis by targeting BACE1, may be one of the presumed targets in AD development, and may provide potential new insights into genetic therapy against $\mathrm{AD}$ ( $\mathrm{Li} \mathrm{H}$ et al.2018). Figure 2 briefly schematizes the association of some other lncRNAs with Alzheimer's disease (Massone et al. 2011).

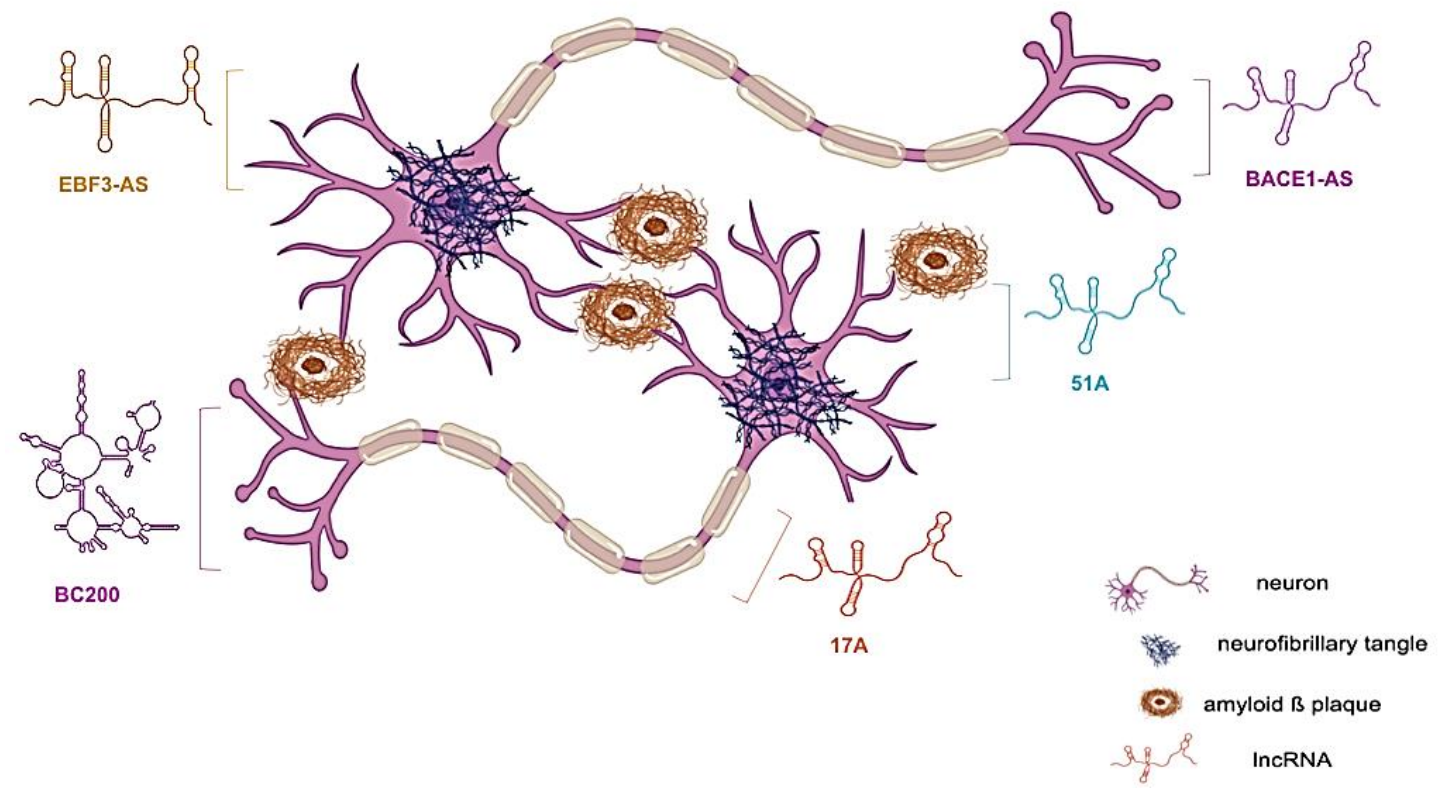

Figure 2. Some lncRNAs associated with AD

\section{Peripheral Blood Mononuclear Cells (PBMCs)}

Mononuclear cells (PBMC) in the peripheral blood are immune system cells consisting of lymphocytes such as T-lymphocytes, B-lymphocytes, natural killer (NK) cells, monocytes, and round nucleus cells such as dendritic cells. They are both myeloid and lymphoid in origin (Ramsay and Gonda 2008). These cells are isolated directly from peripheral blood. Cell fractions containing erythrocytes and granulocytes (neutrophils, basophils, eosinophils) can be easily separated from whole blood by density-graded centrifugation. The medium with a density of $1.077 \mathrm{~g} / \mathrm{ml}$ divides the whole blood into two fractions; PBMCs form the population of cells that remain in the higher phase, while erythrocytes remain in the higher density lower phase. PBMCs have recently been of interest to identify biomarkers of neurodegeneration and to share most of the nonsaptic biochemical environment of neurons.

Studies have shown that $\mathrm{AD}$ is characterized by multiple dysregulation, including sensitivity to apoptosis at PBMC level. Since apoptosis may be the main neuronal death type in AD, apoptotic changes in 
lymphocytes from Alzheimer patients in response to apoptotic stimuli have been shown to be detected simultaneously (Eckert et al 2001), and studies have also been conducted to reflect important processes in the pathogenesis of AD (Tacconi et al 2004, Cosentino et al 2009). Other main findings in PBMCs of Alzheimer's patients include; decreased acetylcholinesterase activity, decreased muscarinic receptor binding, increased oxidative stress, irregular homeostasis of $\mathrm{Ca}++$ concentrations, neurotransmitter receptor expression changes similar to those seen in neurons in the nervous system (Cosentino et al 2009). It has been reported that PBMC platelets are the main source of amyloid peptides in blood plasma $(\sim 90 \%)$ and are similar in structure to amyloid plaque components in Alzheimer's patients and intensify inflammation and increase AD progression (Kucheryavykh et al 2017, Pluta et al 2018). Besides platelets, PBMC lymphocytes are thought to reflect the pathogenesis of $\mathrm{AD}$. For example, impaired $\mathrm{Ca} 2+$ homeostasis and endoplasmic reticulum stress are frequent changes in both AD brain tissue and lymphocytes (Wojsiat et al 2015). Increased amount of reactive oxygen species, defective activities of antioxidant enzymes, mitochondrial sensitivity, DNA damage and apoptosis were also detected in AD lymphocytes (Wojda 2016). This suggests that AD lymphocytes reflect the oxidative stress reaction typical for AD brains. Our in our study (Kurt and Tomatır, 2018); when lncRNA of peripheral blood mononuclear cells is evaluated; significant association of lncRNAs with some metabolic pathways was determined. These include the TNF signaling pathway, PI3K / AKT, Ras, MAPK pathways; glutamergic, dopaminergic, cholinergic synapses; GABA and neurotrophin signaling pathways.

\section{Conclusions}

Recently Alzheimer's disease have been dramatically increase and there is no efficent cure for disease yet. The importance of IncRNAs in basic cellular processes include epigenetic, transcriptional, posttranscriptional and regulating gene expression emphasized today. Some tissue-specific lncRNA expression changes in peripheral blood mononuclear cells may reflect patogenesis of neurodegenerative diseases and help diagnosis and treatment. On the other hand, further studies are needed to determine especially whether processes related to apoptosis pathways are activated in AD.

\section{References}

Akkaya ZY, Dinçer P (2013). Tedavi yaklaşımlarında yeni bir dönem: Kodlamayan RNA'lar ve hastalıklar. $\boldsymbol{M M J} ; 26: 5-10$.

Akman HB, Bensan AE (2014). Noncoding RNAs and cancer. Turk J Biol ;38(6):817-828.

Ayers D (2013). Long Non-Coding RNAs: Novel Emergent Biomarkers for Cancer Diagnostics. Journal of Cancer Research and Treatment;1(2):31-35.

Ayupe AC, Tahira AC, Camargo L, Beckedorff FC, Verjovski-Almeida S, Reis EM (2015). Global analysis of biogenesis, stability and sub-cellular localization of lncRNAs mapping to intragenic regions of the human genome. $\boldsymbol{R N A} \mathbf{B i o l} ; 12(8): 877-92$.

Bär C, Chatterjee S, Thum T (2016). Long Noncoding RNAs in Cardiovascular Pathology, Diagnosis, and Therapy. Circulation;134(19):1484-1499.

Batista PJ, Chang HY (2013). Long noncoding RNAs: cellular address codes in development and disease. Cell; 152:1298-1307.

Bayoğlu B, Cengiz M (2017). The Roles of the Long Non-Coding RNA Transcripts in Cardiovascular Diseases. Bezm Science; 5:74-9.

Beckedorff FC, Amaral MS, Deocesano-Pereira C, Verjovski-Almeida S (2013). Long non-coding RNAs and their implications in cancer epigenetics. Biosci Rep;33(4).

Chew CL, Conos SA, Unal B, Tergaonkar V (2018). Noncoding RNAs: Master Regulators of Inflammatory Signaling. Trends Mol Med (1):66-84. 
Cosentino M, Colombo C, Mauri M, Ferrari M, Corbetta S, Marino F, Bono G, Lecchini S (2009). Expression of apoptosis-related proteins and of mRNA for dopaminergic receptors in peripheral blood mononuclear cells from patients with Alzheimer disease. Alzheimer Dis Assoc Disord;23(1):88-90.

Cummings JL (2004). Alzheimer's disease. N Engl J Med; 351:56.

Derrien T, Johnson R, Bussotti G, Tanzer A, Djebali S, Tilgner H, Guernec G, Martin D, Merkel A, Knowles DG, Lagarde J, Veeravalli L, Ruan X, Ruan Y, Lassmann T, Carninci P, Brown JB, Lipovich L, Gonzalez JM, Thomas M, Davis CA, Shiekhattar R, Gingeras TR, Hubbard TJ, Notredame C, Harrow J, Guigó R (2012). The GENCODE v7 catalog of human long noncoding RNAs: analysis of their gene structure, evolution, and expression. Genome Res; 22:1775-1789.

Eckert A, Oster M, Zerfass R, Hennerici M, Müller WE (2001). Elevated levels of fragmented DNA nucleosomes in native and activated lymphocytes indicate an enhanced sensitivity to apoptosis in sporadic Alzheimer's disease. Specific differences to vascular dementia. Dement Geriatr Cogn Disord;12(2):98-105.

Elkouris M, Kouroupi G, Vourvoukelis A, Papagiannakis N, Kaltezioti V, Matsas R, Stefanis L, Xilouri M, Politis PK (2019). Long Non-coding RNAs Associated With NeurodegenerationLinked Genes Are Reduced in Parkinson's Disease Patients. Front Cell Neurosci.; 13:58.

Fotuhi SN, Khalaj-Kondori M, Hoseinpour Feizi MA, Talebi M (2019). Long Non-coding RNA BACE1-AS May Serve as an Alzheimer's Disease Blood-Based Biomarker. J Mol Neurosci.;10.1007/s12031-019-01364-2.

Gayen S, Maclary E, Hinten M, Kalantry S (2016). Sex-specific silencing of X-linked genes by Xist RNA. Proc Natl Acad Sci U S A;113(3): E309-18. doi: 10.1073/pnas.1515971113.

Gomes CPC, de Gonzalo-Calvo D, Toro R, Fernandes T, Theisen D, Wang DZ, Devaux Y (2018). Non-coding RNAs and exercise: pathophysiological role and clinical application in the cardiovascular system. Clin Sci (Lond) ;132(9):925-942.

Gu C, Chen C, Wu R, Dong T, Hu X, Yao Y, Zhang Y (2018). Long Noncoding RNA EBF3-AS Promotes Neuron Apoptosis in Alzheimer's Disease. DNA Cell Biol;37(3):220-226.

Fallah H, Azari I, Neishabouri MS, Oskooei VK, Taheri M, Ghafouri-Fard S (2019). Sex-specific upregulation of lncRNAs in peripheral blood of patients with schizophrenia. Sci Rep;9(1):12737.

Huang C, Liu S, Wang H, Zhang Z, Yang Q, Gao F (2016). LncRNA PVT1 overexpression is a poor prognostic biomarker and regulates migration and invasion in small cell lung cancer. Am J Transl Res.;8(11):5025-5034.

Iwatsubo T, Odaka A, Suzuki N (1994). Visualization of A beta 42(43) and A beta 40 in senile plaques with end-specific A beta monoclonals: evidence that an initially deposited species is A beta 42(43). Neuron; 13:45-53.

Janika Viereck, Thomas Thum (2017). Circulating Noncoding RNAs as Biomarkers of Cardiovascular Disease and Injury. Circ Res; 120:381-399.

Jazvinšćak JM, Slade N, Hof PR, Šimić G (2018). The interactions of p53 with tau and Aß as potential therapeutic targets for Alzheimer's disease. Prog Neurobiol; S0301-0082(17)30188-0.

Kapranov P, Cheng J, Dike S, Nix DA, Duttagupta R, Willingham AT, Stadler PF, Hertel J, Hackermüller J, Hofacker IL, Bell I, Cheung E, Drenkow J, Dumais E, Patel S, Helt G, Ganesh M, Ghosh S, Piccolboni A, Sementchenko V, Tammana H, Gingeras TR (2007). RNA maps reveal new RNA classes and a possible function for pervasive transcription. Science ; 316: 14841488 . 
Kurt S, Tomatir AG (2018). Alzheimer Hastalığında Long Noncoding RNA (lncRNA) Profillerinin Araştırılması. Unpublished thesis of master of science, Pamukkale University, Institution of Medical Sciences, 91 pages.

Kucheryavykh LY, Dávila-Rodríguez J, Rivera-Aponte DE, Zueva LV, Washington AV, Sanabria P, Inyushin MY (2017). Platelets are responsible for the accumulation of $\beta$-amyloid in blood clots inside and around blood vessels in mouse brain after thrombosis. Brain Res Bull; 128:98-105.

Lashley T, Schott JM, Weston P, Murray CE, Wellington H, Keshavan A, Foti SC, Foiani M, Toombs J, Rohrer JD, Heslegrave A, Zetterberg H (2018). Molecular biomarkers of Alzheimer's disease: progress and prospects. Dis Model Mech;11(5).

Li H, Zheng L, Jiang A, Mo Y, Gong Q (2018). Identification of the biological affection of long noncoding RNA BC200 in Alzheimer's disease. Neuroreport. ;29(13):1061-1067.

Liu W, Zhao J, Lu G (2016). miR-106b inhibits tau phosphorylation at Tyr18 by targeting Fyn in a model of Alzheimer's disease. Biochem Biophys Res Commun;478(2):852-7.

Luo Q, Chen Y (2016). Long noncoding RNAs and Alzheimer's disease. Clin Interv Aging; 11:867872.

Massone S, Vassallo I, Fiorino G (2011). 17A, a novel non-coding RNA, regulates GABA B alternative splicing and signaling in response to inflammatory stimuli and in Alzheimer disease. Neurobiol Dis; 41(2):308-317.

McKhann GM, Knopman DS, Chertkow H, Hyman BT, Jack CR Jr, Kawas CH, Klunk WE, Koroshetz WJ, Manly JJ, Mayeux R, Mohs RC, Morris JC, Rossor MN, Scheltens P, Carrillo MC, Thies B, Weintraub S, Phelps CH (2011). The diagnosis of dementia due to Alzheimer's disease: recommendations from the National Institute on Aging-Alzheimer's Association workgroups on diagnostic guidelines for Alzheimer's disease. Alzheimers Dement;7(3):263-269.

Melissari MT, Grote P (2016). Roles for long non-coding RNAs in physiology and disease. Pflugers Arch;468(6):945-58.

Perez P, Jang SI, Alevizos I (2014). Emerging landscape of non-coding RNAs in oral health and disease. Oral Dis ;20(3):226-35.

Poorkaj P, Bird TD, Wijsman E, Nemens E, Garruto RM, Anderson L, Andreadis A, Wiederholt WC, Raskind M, Schellenberg GD (1998). Tau is a candidate gene for chromosome 17 frontotemporal dementia. Ann Neurol;43(6):815-25.

Prendecki M, Florczak-Wyspianska J, Kowalska M, Ilkowski J, Grzelak T, Bialas K, Kozubski W, Dorszewska J (2019). APOE genetic variants and apoE, miR-107 and miR-650 levels in Alzheimer's disease. Folia Neuropathol. ;57(2):106-116.

Prinz, Kapeller A, Pichler M, Klec C (2019). The Implications of the Long Non-Coding RNA NEAT1 in Non-Cancerous Diseases. Int J Mol Sci.;20(3).

Quinn JJ, Chang HY (2016). Unique features of long non-coding RNA biogenesis and function. Nat Rev Genet;17(1):47-62.

Ramsay RG, Gonda TJ (2008). MYB function in normal and cancer cells. Nat Rev Cancer;8(7):52334.

Reddy PH, Tonk, Kumar S, Vijayan M, Kandimalla R, Kuruva CS, Reddy AP (2017). A critical evaluation of neuroprotective and neurodegenerative MicroRNAs in Alzheimer's disease. Biochem Biophys Res Commun;483(4):1156-1165. 
Ryan P, Patel B, Makwana V, Jadhav HR, Kiefel M, Davey A, Reekie TA, Rudrawar S, Kassiou M (2018). Peptides, Peptidomimetics, and Carbohydrate-Peptide Conjugates as Amyloidogenic Aggregation Inhibitors for Alzheimer's Disease. ACS Chem Neurosci;1023241.

Santoro M, Nociti V, Lucchini M, De Fino C, Losavio FA, Mirabella M (2016). Expression Profile of Long Non-Coding RNAs in Serum of Patients with Multiple Sclerosis. $\boldsymbol{J}$ Mol Neurosci.;59(1):18-23.

Schmitz SU, Grote P, Herrmann BG (2016). Mechanisms of long noncoding RNA function in development and disease. Cell Mol Life Sci;73, 2491-2509.

Selekler K (2010). Alois Alzheimer and Alzheimer's Disease. Turkish Journal of Geriatrics.,13 (914).

Tacconi S, Perri R, Balestrieri E, Grelli S, Bernardini S, Annichiarico R, Mastino A, Caltagirone C, Macchi B (2004). Increased caspase activation in peripheral blood mononuclear cells of patients with Alzheimer's disease. Exp Neurol;190(1):254-62.

Tan L, Yu JT, Hu N, Tan L (2013). Non-coding RNAs in Alzheimer's disease. Mol Neurobiol;47(1):382-93.

Ulitsky I. Interactions between short and long noncoding RNAs. FEBS Lett 2018;1873-3468.

Van Cauwenberghe C, Van Broeckhoven C, Sleegers K (2016). The genetic landscape of Alzheimer disease: clinical implications and perspectives. Genet Med;18(5):421-30.

Wang H, Lu B, Chen J (2019). Knockdown of lncRNA SNHG1 attenuated A 325 -35-inudced neuronal injury via regulating KREMEN1 by acting as a ceRNA of miR-137 in neuronal cells. Biochem Biophys Res Commun.;518(3):438-444.

Wojda U (2016). Alzheimer's disease lymphocytes: potential for biomarkers? Biomark Med; 10:14.

Wojsiat J, Prandelli C, Laskowska-Kaszub K, Mart.n Requero A, Wojda U (2015). Oxidative stress and aberrant cell cycle in Alzheimer's disease lymphocytes: diagnostic prospects. J Alzheimer's Dis; 46:329-350.

Wu G, Cai J, Han Y, Chen J, Huang ZP, Chen C, Cai Y, Huang H, Yang Y, Liu Y, Xu Z, He D, Zhang X, Hu X, Pinello L, Zhong D, He F, Yuan GC, Wang DZ, Zeng C (2014). LincRNA-p21 regulates neointima formation, vascular smooth muscle cell proliferation, apoptosis, and atherosclerosis by enhancing p53 activity. Circulation; 130:1452-1465.

Yang Q, Zhao Q, Yin Y (2019). miR-133b is a potential diagnostic biomarker for Alzheimer's disease and has a neuroprotective role. Exp Ther Med. 18(4):2711-2718.

Yang TT, Liu CG, Gao SC, Zhang Y, Wang PC (2018). The Serum Exosome Derived MicroRNA135a, -193b, and -384 Were Potential Alzheimer's Disease Biomarkers. Biomed Environ Sci;1(2): $87-96$.

Zhang H, Chen Z, Wang X, Huang Z, He Z, Chen Y (2013). Long non-coding RNA: a new player in cancer. J Hematol OncolJ Hematol Oncol;6(1):37. 